Article

\title{
Minimal Wave Speed in a Competitive Integrodifference System without Comparison Principle
}

\author{
Luping Li $* \mathbb{1}$, Shugui Kang, Lili Kong and Huiqin Chen \\ School of Mathematics and Statistics, Shanxi Datong University, Datong 037009, China \\ * Correspondence: sjliluping@sxdtdx.edu.cn or 1lp12309845876@163.com
}

Received: 20 February 2019; Accepted: 21 June 2019; Published: 26 June 2019

\begin{abstract}
We investigate the traveling wave solutions of a competitive integrodifference system without comparison principle. In the earlier conclusions, a threshold of wave speed is defined while the existence or nonexistence of traveling wave solutions remains open when the wave speed is the threshold. By constructing generalized upper and lower solutions, we confirm the existence of traveling wave solutions when the wave speed is the threshold. Our conclusion completes the known results and shows the different decay behavior of traveling wave solutions compared with the case of large wave speeds.
\end{abstract}

Keywords: upper-lower solutions; non-monotone system; minimal wave speed

\section{Introduction}

Spatial propagation thresholds of population models have attracted much attention since these thresholds may characterize the spatial expansion of individuals [1,2], and such a constant invasion speed is coincident with some history data; see some examples by Murray ([1], Chapter 13). For monotone semiflows, some sharp conclusions have been established in the past decades $[3,4]$. In systems admitting a proper comparison principle, some results have also been given, e.g., in competitive systems [4-6] and predator-prey systems [7-9]. In many works, the propagation dynamics were formulated by traveling wave solutions, and minimal wave speed and spreading speeds are important thresholds that have been widely studied.

However, in many models, it is difficult to establish a standard comparison principle due to different reasons, including time delay [10]. When the temporal variable is discrete, the deficiency of monotonicity is also universal, e.g., the discrete logistic model may lead to rich dynamics ([11], Chapter 2). When spatial propagation dynamics are considered, we may refer to some results on the propagation dynamics of non-monotone integrodifference systems by Hsu and Zhao [12], Li et al. [13], Lin [14], Pan and Lin [15], Pan and Zhang [16], and very recent papers [17,18] and references cited therein for other non-monotone diffusion systems. In these works, to establish the minimal wave speed, a general recipe is to pass to a limit function from the results of large wave speeds. The methodology is admissible in scalar equations and irreducible systems since the irreducible leads to proper synchronism, e.g., different components have the same spreading speed in irreducible monotone systems [3]. When reducible system are involved, Lin et al. [19] proved that different species may have different spreading speeds, so it is difficult to establish some thresholds by passing to a limit function. 
In Li and Li [20,21], Pan and Lin [22], and Pan and Liu [23], the authors studied the traveling wave solutions of the following integrodifference system;

$$
\left\{\begin{array}{l}
X_{n+1}(x)=\int_{\mathbb{R}} X_{n}(y) e^{r_{1}\left(1-X_{n}(y)-a Y_{n}(y)\right)} k_{1}(x-y) d y \\
Y_{n+1}(x)=\int_{\mathbb{R}} Y_{n}(y) e^{r_{2}\left(1-Y_{n}(y)-b X_{n}(y)\right)} k_{2}(x-y) d y
\end{array}\right.
$$

in which $n \in \mathbb{N} \bigcup\{0\}$ is a constant, $r_{1}>0, r_{2}>0$ are constants, $a \geq 0, b \geq 0$ are constants describing the interspecific competition, $X_{n}(x), Y_{n}(x)$ denote the densities of two competitors at time $n$ at location $x$ in population dynamics, respectively, and $k_{i}, i=1,2$, are probability functions describing the spatial dispersal of individuals. Firstly, the corresponding difference systems of (1) have been studied by many researchers, including Hofbauer et al. [24], who may model the interspecific and intraspecific competition similar to the famous Lotka-Volterra ordinary differential system ([24], p. 554). Clearly, by taking different parameters in the corresponding difference systems of (1), we may observe different persistence or extinction results, which shows the role of strong or weak interspecific competition [24]. Moreover, the spatial factor was derived from the random movement of individuals and has been widely investigated since the pioneering work of Weinberger [25], which models the spatial-temporal evolutionary of two competitors without the overlapping of generations. Clearly, $(0,0)$ is a trivial steady state of (1), and the corresponding linearized system at $(0,0)$ reads as

$$
X_{n+1}(x)=\int_{\mathbb{R}} X_{n}(y) e^{r_{1}} k_{1}(x-y) d y, Y_{n+1}(x)=\int_{\mathbb{R}} Y_{n}(y) e^{r_{2}} k_{2}(x-y) d y,
$$

which is reducible since $X_{n}, Y_{n}$ are independent of each other.

In this paper, a traveling wave solution of (1) is a special solution taking the form

$$
X_{n}(x)=\phi(\xi), Y_{n}(x)=\psi(\xi), \xi=x+c n \in \mathbb{R},
$$

where $(\phi, \psi)$ is the wave profile and $c>0$ is the wave speed, and so $(\phi, \psi), c$ satisfies

$$
\left\{\begin{array}{l}
\phi(\xi+c)=\int_{\mathbb{R}} \phi(y) e^{r_{1}(1-\phi(y)-a \psi(y))} k_{1}(\xi-y) d y \\
\psi(\xi+c)=\int_{\mathbb{R}} \psi(y) e^{r_{2}(1-\psi(y)-b \phi(y))} k_{2}(\xi-y) d y
\end{array}\right.
$$

for all $\xi \in \mathbb{R}$. With a different asymptotic behavior of $(\phi, \psi)$, a positive solution of (3) may formulate different biological processes. In particular, Pan and Lin [22] considered the solution of (3) satisfying

$$
\lim _{\xi \rightarrow-\infty}(\phi(\xi), \psi(\xi))=(0,0), \liminf _{\xi \rightarrow \infty} \phi(\xi)>0, \liminf _{\xi \rightarrow \infty} \psi(\xi)>0,
$$

which may formulate the invasion of two competitors. They defined a positive threshold $c^{*}$ as follows:

$$
c^{*}=\max \left\{\inf _{\lambda>0} \frac{\ln \left(e^{r_{1}} \int_{\mathbb{R}} e^{\lambda y} k_{1}(y) d y\right)}{\lambda}, \inf _{\lambda>0} \frac{\ln \left(e^{r_{2}} \int_{\mathbb{R}} e^{\lambda y} k_{2}(y) d y\right)}{\lambda}\right\}
$$

under proper convergence conditions on $k_{1}, k_{2}$, and proved the existence of traveling wave solutions if $c>c^{*}$, and the nonexistence of traveling wave solutions if $c \in\left(0, c^{*}\right)$. Moreover, (3) with any given $c>0$ has a positive solution satisfying

$$
\lim _{\xi \rightarrow-\infty}(\phi(\xi), \psi(\xi))=(0,0)
$$

Pan and Lin ([22], Section 4) also investigated the limit behavior of $\xi \rightarrow \infty$. However, it remains open on the existence or nonexistence of (3)-(4) if $c=c^{*}$.

From [22], $c^{*}$ is the potential minimal wave speed, and the purpose of this paper is to further investigate the existence or nonexistence of (3)-(4) if $c=c^{*}$. By ([22], Section 4), it suffices to confirm 
the existence or nonexistence of (3) if $c=c^{*}$. To finish the job, we cannot use the recipes for monotone systems or scalar equations by passing to a limit function from the property of (2) and shall utilize the abstract conclusions in Lin [14] and the methods in Li et al. [26]. However, there are also some significant differences between (1) and the model in ([14], Section 5) or Li et al. [26]. More precisely, the model in [26] is monotone such that the classical upper and lower solutions are applicable, but the model in this paper is not monotone. In fact, considering $x e^{r(1-x)}, r>0$ with

$$
L=\left\{\begin{array}{l}
1, r \in(0,1], \\
e^{r-1} / r(>1), r>1,
\end{array}\right.
$$

we see that $[0, L]$ is the invariant interval of difference equation $u_{n+1}=u_{n} e^{r\left(1-u_{n}\right)}$, but $x e^{r(1-x)}, x \in[0, L]$ with $r>1$ is not monotone since $1 / r<u_{n} \leq u_{n+1} \leq L$ for some $n \in \mathbb{N}$ leads to $u_{n+2} \leq u_{n+1}$. For coupled system (1), the non-monotonicity is also clear if $r_{1}>1$ or $r_{2}>1$.

In [22], the authors proved that if $c>c^{*}$, then (3) has a positive solution satisfying (5), and both $\phi, \psi$ decay exponentially as $\xi \rightarrow-\infty$. In this paper, motivated by [14,26], we construct proper generalized upper and lower solutions to prove the existence of traveling wave solutions if the wave speed $c=c^{*}$, which may complete the conclusion in [22]. Moreover, our result implies that at least one component of $\phi, \psi$ does not decay exponentially when the wave speed $c=c^{*}$ and $\xi \rightarrow-\infty$.

\section{Preliminaries}

In this paper, we shall use the standard partial ordering in $\mathbb{R}^{2}$. That is, if

$$
u=\left(u_{1}, u_{2}\right), v=\left(v_{1}, v_{2}\right) \in \mathbb{R}^{2},
$$

then

$$
u \leq v \text { if and only if } u_{1} \leq v_{1}, u_{2} \leq v_{2} .
$$

The functional space $X$ will be defined by

$$
X=\left\{u(x): \mathbb{R} \rightarrow \mathbb{R}^{2} \text { is bounded and uniformly continuous }\right\} .
$$

By the property of function $x e^{-x}$, we define two constants

$$
L_{i}=\left\{\begin{array}{l}
1, r_{i} \leq 1, \\
\frac{e^{r_{i}-1}}{r_{i}}, r_{i}>1
\end{array} \quad \text { for } i=1,2 .\right.
$$

Then for $i=1,2$, we have

$$
u e^{r_{i}(1-u)}:\left[0, L_{i}\right] \rightarrow\left[0, L_{i}\right]
$$

which implies the positive invariance of the difference equation $u_{n+1}=u_{n} e^{r_{i}\left(1-u_{n}\right)}$ and (1).

In this paper, we assume that $k_{1}, k_{2}$ satisfy

(K) For any $i \in\{1,2\}, k_{i}(x) \in X$ admits compact support, $k_{i}(x)=k_{i}(-x)$, and $\int_{\mathbb{R}} k_{i}(x) d x=1$.

Once $(\mathrm{K})$ holds, it is evident that $c^{*}$ is well defined and is bounded. From the definition of $c^{*}$, we also denote

$$
c_{1}^{*}=\inf _{\lambda>0} \frac{\ln \left(e^{r_{1}} \int_{\mathbb{R}} e^{\lambda y} k_{1}(y) d y\right)}{\lambda}, c_{2}^{*}=\inf _{\lambda>0} \frac{\ln \left(e^{r_{2}} \int_{\mathbb{R}} e^{\lambda y} k_{2}(y) d y\right)}{\lambda},
$$

for the sake of convenience. By simple analysis, we have the following conclusion.

Lemma 1. For any given $i \in\{1,2\}$, we define

$$
\Lambda_{i}(\lambda, c)=\int_{\mathbb{R}} e^{r_{i}+\lambda y-\lambda c} k_{i}(y) d y, \lambda>0, c>0 .
$$


Then $\Lambda_{i}$ satisfies the following items:

(1) If $c \in\left(0, c_{i}^{*}\right)$, then $\Lambda_{i}(\lambda, c)>1, \lambda>0$.

(2) $\Lambda_{i}\left(\lambda, c_{i}^{*}\right) \geq 1, \lambda>0$, and $\Lambda_{i}\left(\lambda, c_{i}^{*}\right)=1$ has a unique solution $\lambda_{i}\left(c_{i}^{*}\right)$ such that

$$
\left.\frac{\partial \Lambda_{i}(\lambda, c)}{\partial \lambda}\right|_{(\lambda, c)=\left(\lambda_{i}\left(c_{i}^{*}\right), c_{i}^{*}\right)}=0
$$

(3) If $c>c_{i}^{*}$ is given, then $\Lambda_{i}\left(\lambda_{i}, c\right)=1$ has two positive roots $0<\lambda_{i 1}(c)<\lambda_{i 2}(c)$ such that $\Lambda_{i}(\lambda, c)<$ $1, \lambda \in\left(\lambda_{i 1}(c), \lambda_{i 2}(c)\right)$.

Proof. Clearly, we have

$$
\lim _{\lambda \rightarrow 0^{+}} \frac{\ln \left(e^{r_{1}} \int_{\mathbb{R}} e^{\lambda y} k_{1}(y) d y\right)}{\lambda}=\infty
$$

and

$$
\lim _{\lambda \rightarrow \infty} \frac{\ln \left(e^{r_{1}} \int_{\mathbb{R}} e^{\lambda y} k_{1}(y) d y\right)}{\lambda}=\infty .
$$

By the continuity of

$$
\frac{\ln \left(e^{r_{1}} \int_{\mathbb{R}} e^{\lambda y} k_{1}(y) d y\right)}{\lambda}, \lambda \in(0, \infty)
$$

and

$$
\ln \left(e^{r_{1}} \int_{\mathbb{R}} e^{y} k_{1}(y) d y\right)<\infty
$$

we see that $c_{1}^{*} \in(0, \infty)$, as for $c_{2}^{*}$. Again by the convex of $\Lambda_{1}(\lambda, c), \Lambda_{2}(\lambda, c)$, we may finish the verification.

To prove the existence of traveling wave solutions, one recipe is to deduce the existence of traveling wave solutions from the existence of upper and lower solutions [12,14,21,27]. In particular, Lin ([14], Theorem 3.5) does not require a monotone condition and may be applied to many systems, so it can be applied to (3). By Lin ([14], Theorem 3.5), we have the following abstract results on the existence of (3).

Lemma 2. Assume that there exist continuous functions $(\underline{\phi}, \underline{\psi}),(\bar{\phi}, \bar{\psi}) \in X$ such that

(1) $(0,0) \leq(\underline{\phi}(\xi), \underline{\psi}(\xi)),(\bar{\phi}(\xi), \bar{\psi}(\xi)) \leq\left(L_{1}, L_{2}\right), \xi \in \mathbb{R}$,

(2) for any given $(\widehat{\phi}, \widehat{\psi}) \in X$ satisfying

$$
(\underline{\phi}(\xi), \underline{\psi}(\xi)) \leq(\widehat{\phi}(\xi), \widehat{\psi}(\xi)) \leq(\bar{\phi}(\xi), \bar{\psi}(\xi)), \xi \in \mathbb{R}
$$

we have

$$
\left\{\begin{array}{l}
\bar{\phi}(\xi+c) \geq \int_{\mathbb{R}} \widehat{\phi}(y) e^{r_{1}(1-\widehat{\phi}(y)-a \widehat{\psi}(y))} k_{1}(\xi-y) d y, \\
\bar{\psi}(\xi+c) \geq \int_{\mathbb{R}} \widehat{\psi}(y) e^{r_{2}(1-\widehat{\psi}(y)-b \widehat{\phi}(y))} k_{2}(\xi-y) d y,
\end{array}\right.
$$

and

$$
\left\{\begin{array}{l}
\underline{\phi}(\xi+c) \leq \int_{\mathbb{R}} \widehat{\phi}(y) e^{r_{1}(1-\widehat{\phi}(y)-a \widehat{\psi}(y))} k_{1}(\xi-y) d y \\
\underline{\psi}(\xi+c) \leq \int_{\mathbb{R}} \widehat{\psi}(y) e^{r_{2}(1-\widehat{\psi}(y)-b \widehat{\phi}(y))} k_{2}(\xi-y) d y
\end{array}\right.
$$

for all $\xi \in \mathbb{R}$. Then (3) has a positive solution $(\phi, \psi) \in X$ such that

$$
(\underline{\phi}(\xi), \underline{\psi}(\xi)) \leq(\phi(\xi), \psi(\xi)) \leq(\bar{\phi}(\xi), \bar{\psi}(\xi)), \xi \in \mathbb{R} .
$$

Remark 1. In the above lemma, $(\underline{\phi}, \underline{\psi}),(\bar{\phi}, \bar{\psi}) \in X$ are a pair of (generalized) upper and lower solutions of (3). 


\section{Main Result}

We first present the main conclusion of this paper as follows.

Theorem 1. Assume that

$$
a L_{2}<1, b L_{1}<1 .
$$

Then (3)-(4) admits a positive solution if and only if $c \geq c^{*}$.

From Pan and Lin ([22], Theorems 4.6 and 5.2), we have the following conclusion.

Lemma 3. If $c>(<) c^{*}$, then (3) has (does not have) a positive solution satisfying (4). Moreover, a positive solution of (3) satisfies (4) if

$$
\phi\left(\xi_{1}\right)>0, \psi\left(\xi_{2}\right)>0
$$

for some $\xi_{1}, \xi_{2} \in \mathbb{R}$.

In what follows, we shall finish the proof of the remainder of Theorem 1 by Lemma 2, that is, the case of $c=c^{*}$, and the first result is established for $c_{1}^{*}>c_{2}^{*}$.

Lemma 4. If $c=c^{*}=c_{1}^{*}>c_{2}^{*}$, then (3)-(4) has a positive solution.

Proof. By Lemmas 2 and 3, it suffices to construct and verify upper and lower solutions of (3) satisfying Lemma 2, which shall confirm the existence of (3) and are motivated by the monotone case in [26].

Firstly, we construct upper and lower solutions, and we need to introduce some parameters. Let $\bar{L}>1$ be large enough such that

$$
\max _{\tilde{\zeta}<0}\left\{-\bar{L} \xi e^{\lambda_{1}^{*}} \xi\right\}>1
$$

With such a constant $\bar{L}$, denote $\xi_{1}$ as the smaller root of

$$
-\bar{L} \xi e^{\lambda_{1}^{c^{*}} \xi}=L_{1}
$$

and $\xi_{2}$ as the larger root of

$$
-\bar{L} \xi e^{\lambda_{1}^{c^{*}} \xi}=L_{1} .
$$

Clearly, if $\bar{L}>1$ is large enough, then $\xi_{2}-\xi_{1}>S+2 c^{*}$, where $S>0$ such that $k_{1}(x)=0,|x|>S$. By these constants, we define continuous functions

$$
\begin{gathered}
\bar{\phi}(\xi)=\left\{\begin{array}{l}
-\bar{L} \xi e^{\lambda_{1}^{*} \xi}, \xi<\xi_{1}, \\
L_{1}, \xi \geq \xi 1,
\end{array}\right. \\
\underline{\phi}(\xi)=\left\{\begin{array}{l}
\max \left\{(-\bar{L} \xi-q \sqrt{-\xi}) e^{\lambda_{1}^{c^{*}} \xi}, 0\right\}, \xi<0, \\
0, \xi \geq 0,
\end{array}\right.
\end{gathered}
$$

and

$$
\begin{aligned}
& \bar{\psi}(\xi)=\min \left\{e^{\lambda_{11}^{c_{1}^{*}} \xi}, L_{2}\right\}, \xi \in \mathbb{R}, \\
& \underline{\psi}(\xi)=\max \left\{e^{\lambda_{21}^{c^{*}} \xi}-q e^{\eta \lambda_{21}^{c^{*}} \xi}, 0\right\}, \xi \in \mathbb{R},
\end{aligned}
$$


where $q>1$ is a constant clarified later, and $\eta \in(1,2)$ satisfies

$$
\lambda_{21}^{c^{*}}<\eta \lambda_{21}^{c^{*}}<\min \left\{\lambda_{22}^{c^{*}}, \lambda_{21}^{c^{*}}+\frac{\lambda_{1}^{c^{*}}}{2}\right\}
$$

such that $\Lambda_{2}\left(\eta \lambda_{21}^{c^{*}}, c^{*}\right)<1$. For fixed constants, the boundedness and uniform continuity of (8)-(11) are evident.

Now, we verify the definition of upper and lower solutions or inequalities (6) and (7). For the first part of (6), the result is clear if $\bar{\phi}\left(\xi+c^{*}\right)=L_{1}$. Otherwise, it suffices to prove that

$$
\int_{\mathbb{R}} \widehat{\phi}(y) e^{r_{1}(1-\widehat{\phi}(y)-a \widehat{\psi}(y))} k_{1}(\xi-y) d y \leq \int_{\mathbb{R}} \widehat{\phi}(y) e^{r_{1}} k_{1}(\xi-y) d y \leq \int_{\mathbb{R}} \bar{\phi}(y) e^{r_{1}} k_{1}(\xi-y) d y \leq \bar{\phi}\left(\xi+c^{*}\right),
$$

since

$$
\begin{aligned}
\int_{\mathbb{R}} \bar{\phi}(y) e^{r_{1}} k_{1}(\xi-y) d y & =\int_{\mathbb{R}} \bar{\phi}(\xi-y) e^{r_{1}} k_{1}(y) d y \\
& \leq-e^{r_{1}} \bar{L} \int_{\mathbb{R}}(\xi-y) e^{\lambda c_{1}^{*}(\xi-y)} k_{1}(y) d y \\
& \leq-e^{r_{1}} \bar{L} \xi e^{\lambda_{1}^{c^{*}} \xi} \int_{\mathbb{R}} e^{-\lambda_{1}^{c^{*}} y} k_{1}(y) d y+e^{r_{1}} \bar{L} e^{\lambda_{1}^{*} \xi} \int_{\mathbb{R}} y e^{-\lambda_{1}^{c^{*}} y} k_{1}(y) d y \\
& =-e^{r_{1}} \bar{L} \bar{\xi} e^{\lambda_{1}^{c^{*}} \xi} \int_{\mathbb{R}} e^{\lambda_{1}^{*} y} k_{1}(y) d y-e^{r_{1}} \bar{L} e^{\lambda_{1}^{*} \xi} \int_{\mathbb{R}} y e^{\lambda_{1}^{c^{*}} y} k_{1}(y) d y \\
& =-\bar{L}\left(\xi+c^{*}\right) e^{\lambda_{1}^{*}\left(\xi+c^{*}\right)}=\bar{\phi}\left(\xi+c^{*}\right),
\end{aligned}
$$

which finishes the verification of the first part of (6).

On the second part of (6), the result is clear if $\bar{\psi}\left(\xi+c^{*}\right)=L_{2}$. Otherwise, $\bar{\psi}\left(\xi+c^{*}\right)=e^{\lambda_{21}^{c_{2}^{*}}\left(\xi+c^{*}\right)}$ and

$$
\bar{\psi}(\xi-y) \leq e^{\lambda c_{21}^{*}(\xi-y)}, y \in \mathbb{R}
$$

such that

$$
\begin{aligned}
\int_{\mathbb{R}} \hat{\psi}(y) e^{r_{2}(1-\hat{\psi}(y)-b \hat{\phi}(y))} k_{2}(\xi-y) d y & \leq \int_{\mathbb{R}} \bar{\psi}(y) e^{r_{2}} k_{2}(\xi-y) d y \\
& \leq e^{r_{2}} \int_{\mathbb{R}} e^{\lambda_{21}^{*}(\xi-y)} k_{2}(y) d y \\
& =e^{\lambda_{21}^{*}\left(\xi+c^{*}\right)}
\end{aligned}
$$

which finishes the verification of (6).

We now present the verification of (7). If $\underline{\phi}\left(\xi+c^{*}\right)=0$, then (7) is clear by

$$
\underline{\phi}(\xi-y) \geq 0, y \in \mathbb{R} .
$$

Otherwise, let $q>q_{1}>\bar{L}$ such that

$$
-\bar{L} \xi-q_{1} \sqrt{-\xi}>0
$$

implies

$$
\underline{\phi}(\xi-y) \leq \bar{\phi}(\xi-y) \leq e^{\lambda^{\prime}(\xi-y)},|y| \leq S,
$$

where

$$
\lambda^{\prime}+\lambda_{21}^{c^{*}}>\lambda_{1}^{c^{*}}, 2 \lambda^{\prime}>\lambda_{1}^{c^{*}}
$$


By the boundedness, there exists a constant $L>0$ such that

$$
e^{-x}>1-L x, x \in\left[0, L_{1}+L_{2}\right],
$$

then we have

$$
\begin{aligned}
& \int_{\mathbb{R}} \hat{\phi}(y) e^{r_{1}(1-\hat{\phi}(y)-a \hat{\psi}(y))} k_{1}(\xi-y) d y \\
\geq & \int_{\mathbb{R}} \underline{\phi}(y) e^{r_{1}(1-\bar{\phi}(y)-a \bar{\psi}(y))} k_{1}(\xi-y) d y \\
\geq & e^{r_{1}} \int_{\mathbb{R}} \underline{\phi}(y)(1-L \bar{\phi}(y)-a L \bar{\psi}(y)) k_{1}(\xi-y) d y \\
= & e^{r_{1}} \int_{\mathbb{R}} \underline{\phi}(\xi-y)(1-L \bar{\phi}(\xi-y)-a L \bar{\psi}(\xi-y)) k_{1}(y) d y \\
\geq & e^{r_{1}} \int_{\mathbb{R}} \underline{\phi}(\xi-y) k_{1}(y) d y-e^{r_{1}} L \int_{\mathbb{R}} \bar{\phi}^{2}(\xi-y) k_{1}(y) d y-e^{r_{1}} a L \int_{\mathbb{R}} \bar{\phi}(\xi-y) \bar{\psi}(\xi-y) k_{1}(y) d y \\
\geq & e^{r_{1}} \int_{\mathbb{R}}\left[(-\bar{L}(\xi-y)-q \sqrt{-(\xi-y)}) e^{\lambda_{1}^{c^{*}}(\xi-y)}\right] k_{1}(y) d y \\
& -e^{r_{1}} L \int_{\mathbb{R}} e^{2 \lambda^{\prime}(\xi-y)} k_{1}(y) d y-e^{r_{1}} a L \int_{\mathbb{R}} e^{\left(\lambda^{\prime}+\lambda_{21}^{c^{*}}\right)(\xi-y)} k_{1}(y) d y .
\end{aligned}
$$

Due to the verification of (6), we only need to confirm that

$$
\begin{aligned}
& q \sqrt{-\left(\xi+c^{*}\right)} e^{\lambda_{1}^{*}\left(\xi+c^{*}\right)} \\
\geq & q e^{r_{1}} \int_{\mathbb{R}} \sqrt{-(\xi-y)} e^{\lambda_{1}^{*}(\xi-y)} k_{1}(y) d y+e^{r_{1}} L \int_{\mathbb{R}} e^{2 \lambda^{\prime}(\xi-y)} k_{1}(y) d y+e^{r_{1}} a L \int_{\mathbb{R}} e^{\left(\lambda^{\prime}+\lambda_{21}^{c^{*}}\right)(\xi-y)} k_{1}(y) d y
\end{aligned}
$$

or

$$
\begin{aligned}
& q\left[\sqrt{-\left(\xi+c^{*}\right)} e^{\lambda_{1}^{*}\left(\xi+c^{*}\right)}-e^{r_{1}} \int_{\mathbb{R}} \sqrt{-(\xi-y)} e^{\lambda_{1}^{*}(\xi-y)} k_{1}(y) d y\right] \\
= & q e^{r_{1}} \int_{\mathbb{R}}\left[\sqrt{-\left(\xi+c^{*}\right)}-\sqrt{-(\xi-y)}\right] e^{\lambda c_{1}^{c^{*}}(\xi-y)} k_{1}(y) d y \\
= & q e^{r_{1}} e^{\lambda_{1}^{c^{*}} \xi} \int_{\mathbb{R}}\left[\sqrt{-\left(\xi+c^{*}\right)}-\sqrt{-(\xi-y)}\right] e^{-\lambda_{1}^{c^{*}} y} k_{1}(y) d y \\
= & q e^{r_{1}} e^{\lambda_{1}^{c^{*} \xi}} \int_{\mathbb{R}}\left[\sqrt{-\left(\xi+c^{*}\right)}-\sqrt{-(\xi+y)}\right] e^{\lambda_{1}^{*} y} k_{1}(y) d y \\
\geq & e^{r_{1}} L \int_{\mathbb{R}} e^{2 \lambda^{\prime}(\xi-y)} k_{1}(y) d y+e^{r_{1}} a L \int_{\mathbb{R}} e^{\left(\lambda^{\prime}+\lambda_{21}^{c^{*}}\right)(\xi-y)} k_{1}(y) d y .
\end{aligned}
$$

By direct calculations, we have

$$
\begin{aligned}
& e^{r_{1}} \int_{\mathbb{R}}\left[\sqrt{-\left(\xi+c^{*}\right)}-\sqrt{-(\xi+y)}\right] e^{\lambda c_{1}^{*} y} k_{1}(y) d y \\
= & e^{r_{1}} \int_{-\infty}^{c^{*}} \frac{\left(y-c^{*}\right) e^{\lambda c_{1}^{*} y}}{\sqrt{-\left(\xi+c^{*}\right)}+\sqrt{-(\xi+y)}} k_{1}(y) d y+e^{r_{1}} \int_{c^{*}}^{\infty} \frac{\left(y-c^{*}\right) e^{\lambda_{1}^{*} y}}{\sqrt{-\left(\xi+c^{*}\right)}+\sqrt{-(\xi+y)}} k_{1}(y) d y .
\end{aligned}
$$

If $y \in\left(-\infty, c^{*}\right]$, then $y-c^{*}<0$ such that

$$
\frac{y-c^{*}}{\sqrt{-\left(\xi+c^{*}\right)}+\sqrt{-(\xi+y)}} \geq \frac{y-c^{*}}{2 \sqrt{-\left(\xi+c^{*}\right)}}
$$


and

$$
\begin{aligned}
& q e^{r_{1}} \int_{\mathbb{R}}\left[\sqrt{-\left(\xi+c^{*}\right)}-\sqrt{-(\xi+y)}\right] e^{\lambda c_{1}^{*} y} k_{1}(y) d y \\
\geq & q e^{r_{1}} \int_{-\infty}^{c^{*}} \frac{y-c^{*}}{2 \sqrt{-\left(\xi+c^{*}\right)}} e^{\lambda_{1}^{*} y} k_{1}(y) d y+q e^{r_{1}} \int_{c^{*}}^{\infty} \frac{y-c^{*}}{\sqrt{-\left(\xi+c^{*}\right)}+\sqrt{-(\xi+y)}} e^{\lambda_{1}^{*} y} k_{1}(y) d y .
\end{aligned}
$$

Moreover, since

$$
\int_{-\infty}^{c^{*}} \frac{y-c^{*}}{2 \sqrt{-\left(\xi+c^{*}\right)}} e^{\lambda_{1}^{*} y} k_{1}(y) d y+\int_{c^{*}}^{\infty} \frac{y-c^{*}}{2 \sqrt{-\left(\xi+c^{*}\right)}} e^{\lambda_{1}^{c^{*}} y} k_{1}(y) d y=0,
$$

then

$$
\begin{aligned}
& q e^{r_{1}} \int_{\mathbb{R}}\left[\sqrt{-\left(\xi+c^{*}\right)}-\sqrt{-(\xi+y)}\right] e^{\lambda_{1}^{c_{1}^{*}} y} k_{1}(y) d y \\
\geq & q e^{r_{1}} \int_{c^{*}}^{\infty} \frac{y-c^{*}}{\sqrt{-\left(\xi+c^{*}\right)}+\sqrt{-(\xi+y)}} e^{\lambda_{1}^{*} y} k_{1}(y) d y-q e^{r_{1}} \int_{c^{*}}^{\infty} \frac{y-c^{*}}{2 \sqrt{-\left(\xi+c^{*}\right)}} e^{\lambda c_{1}^{*} y} k_{1}(y) d y \\
= & q e^{r_{1}} \int_{c^{*}}^{\infty}\left[\frac{\left(y-c^{*}\right)\left[\sqrt{-\left(\xi+c^{*}\right)}-\sqrt{-(\xi+y)}\right]}{2 \sqrt{-\left(\xi+c^{*}\right)}\left[\sqrt{-\left(\xi+c^{*}\right)}+\sqrt{-(\xi+y)}\right]}\right] e^{\lambda_{1}^{*} y} k_{1}(y) d y \\
= & q e^{r_{1}} \int_{c^{*}}^{\infty}\left[\frac{\left(y-c^{*}\right)^{2}}{2 \sqrt{-\left(\xi+c^{*}\right)}\left[\sqrt{-\left(\xi+c^{*}\right)}+\sqrt{-(\xi+y)}\right]^{2}}\right] e^{\lambda_{1}^{c^{*}} y} k_{1}(y) d y \\
\geq & \frac{q e^{r_{1}}}{8(-\xi)^{\frac{3}{2}}} \int_{c^{*}}^{\infty}\left(y-c^{*}\right)^{2} e^{\lambda_{1}^{*} y} k_{1}(y) d y .
\end{aligned}
$$

So, the result is true if

$$
\begin{aligned}
& \frac{q e^{r_{1}} e^{\lambda_{1}^{1^{*}} \xi}}{8(-\xi)^{\frac{3}{2}}} \int_{\mathcal{c}^{*}}^{\infty}\left(y-c^{*}\right)^{2} e^{\lambda_{1}^{c^{*}} y} k_{1}(y) d y \\
\geq & L e^{r_{1}} \int_{\mathbb{R}} e^{2 \lambda^{\prime}(\xi-y)} k_{1}(y) d y+L a e^{r_{1}} \int_{\mathbb{R}} e^{\left(\lambda /+\lambda_{21}^{c_{1}^{*}}\right)(\xi-y)} k_{1}(y) d y,
\end{aligned}
$$

which holds by letting $q>1$ be large enough. This completes the verification of the first part of (7).

We now consider the second part of (7), and it is clear if $\underline{\psi}\left(\xi+c^{*}\right)=0$. Otherwise, let $q>1$ be large such that $\underline{\psi}(\xi)>0$ implies

$$
\bar{\phi}(\xi)<e^{\lambda_{1}^{c} \xi / 2}
$$

By the monotonicity, we may finish the verification of (7) if

$$
\begin{aligned}
& \int_{\mathbb{R}} \widehat{\psi}(y) e^{r_{2}(1-\widehat{\psi}(y)-b \hat{\phi}(y))} k_{2}(\xi-y) d y \\
\geq & \int_{\mathbb{R}} \underline{\psi}(y) e^{r_{2}(1-\bar{\psi}(y)-b \bar{\phi}(y))} k_{2}(\xi-y) d y \\
\geq & e^{r_{2}} \int_{\mathbb{R}} \underline{\psi}(y) k_{2}(\xi-y) d y-L e^{r_{2}} b \int_{\mathbb{R}} \bar{\psi}(y)[\bar{\psi}(y)+b \bar{\phi}(y)] k_{2}(\xi-y) d y \\
\geq & \underline{\psi}(\xi+c) .
\end{aligned}
$$

By direct calculation, the above inequality holds if

$$
q>\frac{e^{r_{2}} L(1+b)\left[\int_{\mathbb{R}} e^{2 \lambda_{21}^{*} y} k_{2}(y) d y+\int_{\mathbb{R}} e^{\left(\lambda_{1}^{c^{*}} / 2+\lambda_{21}^{c^{*}}\right) y} k_{2}(y) d y\right]}{1-e^{r_{2}} \int_{\mathbb{R}} e^{\eta \lambda_{21}^{c^{*}}\left(y-c^{*}\right)} k_{2}(y) d y}+1 .
$$


Since we obtain a pair of upper and lower solutions, the proof is complete.

In a similar way, we can obtain the existence of (3)-(4) in two other cases: $c_{2}^{*}<c_{1}^{*}, c_{2}^{*}=c_{1}^{*}$. By what we have done, we confirm that $c^{*}$ is the threshold such that (3)-(4) has a positive solution, which completes the proof of Theorem 1 . Before ending this section, we point out that it is easy to verify the conditions $a L_{1}<1, b L_{2}<1$. For example, with any given $r_{2}>0, a>0$, we present the following numerical result (Figure 1).

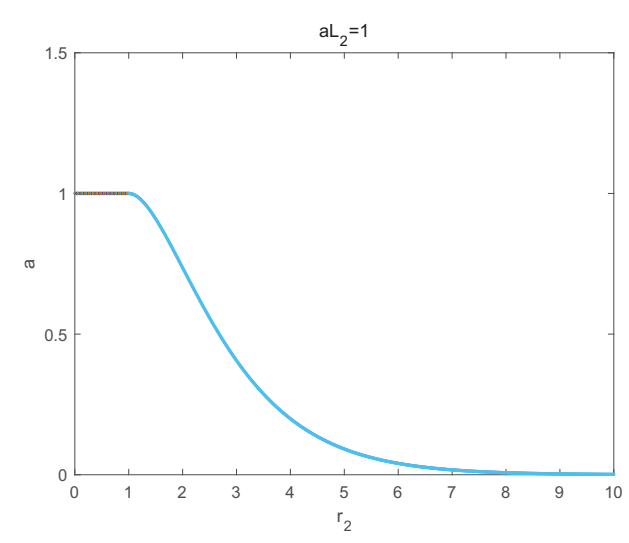

Figure 1. Under the line, $a L_{2}<1$.

\section{Concluding Remarks}

A traveling wave solution is a special entire solution, and similar propagation phenomena have been widely observed in different fields ([28], Chapter 1). The minimal wave speed of traveling wave solutions is important since it may characterize the propagation threshold, and it has been widely studied for monotone systems. Since traveling wave solutions involve long-term behavior, it is difficult to show general results for non-monotone reducible systems, including (1). In this paper, we obtain the minimal wave speed of (1), and the corresponding traveling wave solutions formulate the coinvasion-coexistence process of two competitors, which is similar to the process of Tang and Fife [6]. On the one hand, our conclusion completes the conclusions in Pan and Lin [22]. On the other hand, we found the decay behavior of traveling wave solutions with a minimal wave speed is different from those with a large wave speed.

In ecological communities, there are many phenomena involving interspecific competition or predator-prey processes, and many non-cooperative or non-monotone systems have been established to model interspecific actions [1,11]. However, non-monotonicity may lead to rich dynamics in these systems, including the occurrence of chaos and bifurcation. Although there are some important records on the spatial propagation of non-cooperative process [2], it is difficult to establish the propagation thresholds in some non-monotone systems. Since we do not require monotonicity in this paper, we hope that our recipe can be applied to more models to formulate the propagation thresholds of multiple species.

Author Contributions: L.L. analyzed and prepared/edited the manuscript, S.K. analyzed and prepared the manuscript, L.K. analyzed and prepared the manuscript, H.C. analyzed and prepared the manuscript. All authors read the final manuscript.

Funding: This work was partially supported by the NSF of China under grant No. 11871314.

Acknowledgments: The authors are grateful to two referees for their careful reading and valuable comments.

Conflicts of Interest: The authors declare no conflict of interest. 


\section{References}

1. Murray, J.D. Mathematical Biology, II. Spatial Models and Biomedical Applications, 3rd ed.; Springer: New York, NY, USA, 2003.

2. Shigesada, N.; Kawasaki, K. Biological Invasions: Theory and Practice; Oxford University Press: Oxford, UK, 1997.

3. Liang, X.; Zhao, X.Q. Asymptotic speeds of spread and traveling waves for monotone semiflows with applications. Commun. Pure Appl. Math. 2007, 60, 1-40. [CrossRef]

4. Weinberger, H.F.; Lewis, M.A.; Li, B. Analysis of linear determinacy for spread in cooperative models. J. Math. Biol. 2002, 45, 183-218. [CrossRef] [PubMed]

5. Lin, G.; Li, W.T. Asymptotic spreading of competition diffusion systems: The role of interspecific competitions. Eur. J. Appl. Math. 2012, 23, 669-689. [CrossRef]

6. Tang, M.M.; Fife, P. Propagating fronts for competing species equations with diffusion. Arch. Ration. Mech. Anal. 1980, 73, 69-77. [CrossRef]

7. Ducrot, A. Spatial propagation for a two component reaction-diffusion system arising in population dynamics. J. Differ. Equ. 2016, 260, 8316-8357. [CrossRef]

8. Lin, G. Invasion traveling wave solutions of a predator-prey system. Nonlinear Anal. 2014, 96, 47-58. [CrossRef]

9. Pan, S. Invasion speed of a predator-prey system. Appl. Math. Lett. 2017, 74, 46-51. [CrossRef]

10. Wu, J. Theory and Applications of Partial Functional Differential Equations; Springer: New York, NY, USA, 1996.

11. Murray, J.D. Mathematical Biology, I. An Introduction, 3rd ed.; Springer: New York, NY, USA, 2002.

12. Hsu, S.B.; Zhao, X.Q. Spreading speeds and traveling waves for nonmonotone integrodifference equations. SIAM J. Math. Anal. 2008, 40, 776-789. [CrossRef]

13. Li, B.; Lewis, M.A.; Weinberger, H.F. Existence of traveling waves for integral recursions with nonmonotone growth functions. J. Math. Biol. 2009, 58, 323-338. [CrossRef]

14. Lin, G. Travelling wave solutions for intefro-difference systems. J. Differ. Equ. 2015, 258, $2908-2940$. [CrossRef]

15. Pan, S.; Lin, G. Propagation of second order integrodifference equations with local monotonicity. Nonlinear Anal. Real World Appl. 2011, 12, 535-544. [CrossRef]

16. Zhang, L.; Pan, S. Entire solutions of integrodifference equations. J. Differ. Equ. Appl. 2019, 25, 504-515. [CrossRef]

17. Li, X.S.; Pan, S. Traveling wave solutions of a delayed cooperative system. Mathematics 2019, 7, 269. [CrossRef]

18. Liu, X.L.; Pan, S. Spreading speed in a nonmonotone equation with dispersal and delay. Mathematics 2019, 7, 291. [CrossRef]

19. Lin, G.; Li, W.T.; Ruan S. Spreading speeds and traveling waves in competitive recursion systems. J. Math. Biol. 2011, 69, 162-201. [CrossRef]

20. Li, K.; Li, X. Asymptotic behavior and uniqueness of traveling wave solutions in Ricker competition system. J. Math. Anal. Appl. 2012, 389, 486-497. [CrossRef]

21. Li, K.; Li, X. Travelling wave solutions in integro-difference competition system. IMA J. Appl. Math. 2013, 78, 633-650. [CrossRef]

22. Pan, S.; Lin, G. Coinvasion-coexistence travelling wave solutions of an integro-difference competition system. J. Differ. Equ. Appl. 2014, 20, 511-525. [CrossRef]

23. Pan, S.; Liu, J. Bistable traveling wave solutions in a competitive recursion system with Ricker nonlinearity. Electron. J. Qual. Theory Differ. Equ. 2014, 7, 1-9. [CrossRef]

24. Hofbauer, J.; Hutson, V.; Jansen, W. Coexistence for systems governed by difference equations of Lotka-Volterra type. J. Math. Biol. 1987, 25, 553-570. [CrossRef]

25. Weinberger, H.F. Long-time behavior of a class of biological model. SIAM J. Math. Anal. 1982, 13, 353-396. [CrossRef]

26. Li, L.P.; Kang, S.G.; Kong, L.L.; Chen, H.Q. Minimal wave speed of a competition integrodifference system. J. Differ. Equ. Appl. 2018, 24, 941-954. [CrossRef] 
27. Wang, H.; Castillo-Chavez, C. Spreading speeds and traveling waves for non-cooperative integro-difference systems. Discret. Contin. Dyn. Syst. Ser. B 2012, 17, 2243-2266. [CrossRef] [PubMed]

28. Volpert, A.I.; Volpert, V.A.; Volpert, V.A. Traveling Wave Solutions of Parabolic Systems, Translations of Mathematical Monographs 140; AMS: Providence, RI, USA, 1994.

(C) 2019 by the authors. Licensee MDPI, Basel, Switzerland. This article is an open access article distributed under the terms and conditions of the Creative Commons Attribution (CC BY) license (http:/ / creativecommons.org/licenses/by/4.0/). 